\title{
The coexistence curve of finite charged nuclear matter
}

J. B. Elliott*, L. G. Moretto*, L. Phair*, G. J. Wozniak*, L. Beaulieu ${ }^{\dagger}$, H. Breuer**, R. G. Korteling ${ }^{\ddagger}$, K. Kwiatkowski ${ }^{\S}$, T. Lefort ${ }^{\mathbb{\sharp}}$, L. Pienkowskill, A. Ruangma ${ }^{\dagger \dagger}$, V. E. Viola ${ }^{\mathbb{I}}$, S. J. Yennello ${ }^{\dagger \dagger}$, S. Albergo ${ }^{\ddagger \ddagger}$, F. Bieser ${ }^{\S \S}$, F. P. Brady ${ }^{\text {IIII }}$, Z. Caccia ${ }^{\ddagger \ddagger}$, D. A. Cebra ${ }^{\text {ITI }}$, A. D. Chacon***, J. L. Chance ${ }^{\mathbb{T I I I}}$, Y. Choi ${ }^{\dagger \dagger \dagger}$, S. Costa ${ }^{\ddagger *}$, M. L. Gilkes ${ }^{\dagger \dagger \dagger}$, J. A. Hauger ${ }^{\dagger \dagger \dagger}$, A. S. Hirsch ${ }^{\dagger \dagger}$, E. L. Hjort ${ }^{\dagger \dagger \dagger}$, A. Insolia ${ }^{\dagger \dagger}$, M. Justice ${ }^{\ddagger \ddagger}$, D. Keane ${ }^{\ddagger+}$, J. C. Kintner ${ }^{\text {IIII }}$, V. Lindenstruth ${ }^{\S \S}$, M. A. Lisa ${ }^{\S \S}$, H. S. Matis ${ }^{\S \S}$, M. McMahan ${ }^{\S \S}$, C. McParland ${ }^{\S \S}$, W. F. J. Müller ${ }^{\S \S}$, D. L. Olson ${ }^{\S \S}$, M. D. Partlan ${ }^{\text {IIII }}$, N. T. Porile ${ }^{\dagger \dagger \dagger}$, R. Potenza ${ }^{\ddagger \ddagger}$, G. Rai ${ }^{\S \S}$, J. Rasmussen $^{\S \S}$, H. G. Ritter ${ }^{\S \S}$, J. Romanski ${ }^{\ddagger \ddagger}$, J. L. Romero ${ }^{\text {IIII }}$, G. V. Russo ${ }^{\ddagger}$, H. Sann ${ }^{\S \S}$, R. P. Scharenberg ${ }^{\dagger \dagger}$, A. Scott ${ }^{\ddagger \ddagger}$, Y. Shao ${ }^{\ddagger \ddagger}$, B. K. Srivastava ${ }^{\dagger \dagger \dagger}$, T. J. M. Symons ${ }^{\S \S}$,

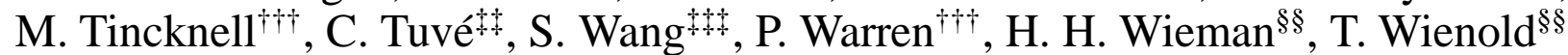
and $\mathrm{K}$. Wolf***

${ }^{*}$ Nuclear Science Division, Lawrence Berkeley National Laboratory, University of California, Berkeley, CA 94720

${ }^{\dagger}$ ISiS Collaboration, Départment de Physique, Université Laval Québec, Canada G1K 7P4

${ }^{* *}$ ISiS Collaboration, Department of Physics, University of Maryland, College Park, MD 20740

${ }^{\ddagger}$ ISiS Collaboration, Department of Chemistry, Simon Fraser University, Burnaby, British Columbia, Canada V5A IS6

${ }^{\S}$ ISiS Collaboration, Los Alamos National Laboratory, Physics Division p-23, Los Alamos, NM 87545

IISiS Collaboration, Department of Chemistry and IUCF, Indiana University, Bloomington, Indiana 47405

"ISiS Collaboration, Heavy Ion Laboratory, Warsaw University, Warsaw, Poland

${ }^{\dagger}$ ISiS Collaboration, Department of Chemistry \& Cyclotron Laboratory, Texas A\&M University, College Station, TX 77843

${ }^{\sharp}$ EOS Collaboration, Universitá di Catania and Istituto Nazionale di Fisica Nucleare-Sezione di Catania, 95129 Catania, Italy

${ }^{\S}$ EOS Collaboration, Nuclear Science Division, Lawrence Berkeley National Laboratory, Berkeley, CA 94720

पIIIEOS Collaboration, University of California, Davis, CA 95616

${ }^{* * *}$ EOS Collaboration, Texas A\&M University, College Station, TX 77843

${ }^{\dagger \dagger}$ EOS Collaboration, Purdue University, West Lafayette, IN 47907

+H EOS Collaboration, Kent State University, Kent, $\mathrm{OH} 44242$

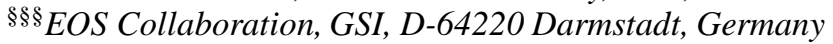

\begin{abstract}
The multifragmentation data of the ISiS Collaboration and the EOS Collaboration are examined. Fisher's droplet formalism, modified to account for Coulomb energy, is used to determine the critical exponents $\tau$ and $\sigma$, the surface energy coefficient $c_{0}$, the pressure-temperature-density coexistence curve of finite nuclear matter and the location of the critical point.
\end{abstract}

This work examines the formation of "fragments" from excited nuclei, termed "nuclear multifragmentation," which may be the result of a liquid-vapor phase transition [1, 2, 3]. Past analyses of nuclear multifragmentation have determined critical exponents [1,4], examined caloric curves [5] and reported negative heat capacities [6]. This work will show that three EOS experimental data sets and the ISiS data set contain a signature of a liquid-vapor phase transition manifested by the scaling behavior of Fisher's droplet formalism. Via Fisher's scaling the coexistence line 
TABLE 1. Fit parameters

\begin{tabular}{llllllll} 
System & $\tau$ & $\sigma$ & $\beta$ & $c_{0}(\mathrm{MeV})$ & $\Delta \mu(\mathrm{AMeV})$ & $x$ & $y$ \\
\hline ISiS $\pi+\mathrm{Au}$ & $2.18 \pm 0.14$ & $0.54 \pm 0.01$ & $0.33 \pm 0.25$ & $18.3 \pm 0.5$ & $0.06 \pm 0.03$ & $1.0 \pm 0.06$ & 1.00 (fixed) \\
EOS Au + C & 2.2 (fixed) & $0.69 \pm 0.02$ & $0.30 \pm 0.01$ & $14 \pm 1$ & $0.38 \pm 0.02$ & $1.0 \pm 0.1$ & $0.43 \pm 0.06$ \\
EOS La + C & 2.2 (fixed) & $0.69 \pm 0.02$ & $0.30 \pm 0.01$ & $14 \pm 1$ & $0.42 \pm 0.03$ & $1.2 \pm 0.1$ & $0.33 \pm 0.08$ \\
EOS Kr + C & 2.2 (fixed) & $0.69 \pm 0.02$ & $0.30 \pm 0.01$ & $14 \pm 1$ & $0.61 \pm 0.05$ & $3.9 \pm 0.7$ & $0.70 \pm 0.20$
\end{tabular}

is observed over a large temperature interval extending up to and including the critical point. Critical exponents $\tau$ and $\sigma$, the critical temperature $T_{c}$, the surface energy coefficient $c_{0}$, the compressibility factor $C_{F}$, the pressure-densitytemperature coexistence curve and a measure of the critical pressure $p_{c}$ and critical density $\rho_{c}$ can be determined.

The Indiana Silicon Sphere (ISiS) Collaboration collected over 1,000,000 events for the reaction $8.0 \mathrm{GeV} / \mathrm{c} \pi+$ $\mathrm{Au}$. For every event the fragment charge distribution was recorded for $1 \leq Z \leq 15$, fragments with $Z>15$ were not elementally resolved [7]. Particles knocked out of the gold nucleus in the projectile-target collision were differentiated from the fragments formed from the excited remnant via a charge dependent kinetic energy cut [8]. An estimate was made of the charge of the fragmenting system $Z_{0}$ by subtracting the charge of the knockout particles from the charge of the gold nucleus. The mass of the fragmenting system $A_{0}$ was estimated by assuming that 1.7 were neutrons knocked from the gold nucleus for every proton. The excitation energy per nucleon of the remnant $E^{*}$ was constructed via energy balance considerations and the data was binned in terms of $E^{*}$ in units of tenth of an AMeV.

The EOS Collaboration collected $\sim 25,000$ fully reconstructed events $\left(76 \leq Z_{\text {observed }} \leq 82\right)$ for the reaction 1.0 $\mathrm{AGeV} \mathrm{Au}+\mathrm{C}, \sim 22,000$ fully reconstructed events $\left(54 \leq Z_{\text {observed }} \leq 60\right)$ for $1.0 \mathrm{AGeV} \mathrm{La}+\mathrm{C}$ and $\sim 36,000$ fully reconstructed events $\left(32 \leq Z_{\text {observed }} \leq 39\right)$ for $1.0 \mathrm{AGeV} \mathrm{Kr}+\mathrm{C}$ [9]. For every event, the charge and mass of the projectile remnant $\left(Z_{0}, A_{0}\right)$ were determined by subtracting the charge and mass of the particles knocked out of the projectile from the charge and mass of the projectile. The knockout particles were distinguished from the fragments via a constant $30 \mathrm{MeV}$ kinetic energy cut and $E^{*}$, constructed via energy balance considerations, was corrected for collective expansion effects [9]. The data for each system was binned in terms of $E^{*}$ in units of half an AMeV.

The basis of the present analysis lies in an examination of the fragment yield distribution in the context of Fisher's droplet formalism [10]. Fisher gives the number of droplets of size $A$ normalized to the size of the system as:

$$
n_{A}(\varepsilon)=q_{0} A^{-\tau} \exp \left(\frac{A \Delta \mu}{T}-\frac{c_{0} \varepsilon A^{\sigma}}{T}\right),
$$

where $\tau$ is the topological critical exponent, for three dimensions $2 \leq \tau \leq 3 ; q_{0}$ is a normalization constant depending solely on $\tau$ [11]; $\Delta \mu=\mu-\mu_{\text {coex }}$ with $\mu$ as the chemical potential of the system and $\mu_{\text {coex }}$ as the chemical potential at coexistence; $T$ is the temperature; $\sigma$ is a critical exponent related to the ratio of the dimensionality of the surface to the volume; $c_{0}$ is the zero temperature surface energy coefficient; $\varepsilon=\left(T_{c}-T\right) / T_{c}$ is a measure of the distance from the critical point; and $T_{c}$ is the critical temperature. This form of the surface energy is applicable only for $T \leq T_{c}$.

The fragment yields were fit to Eq. (1) modified to account for the Coulomb energy when a fragment moves from the liquid to the vapor (à la fission):

$$
n_{A}=q_{0} A^{-\tau} \exp \left(\frac{A \Delta \mu+E_{C o u l}}{T}-\frac{c_{0} \varepsilon A^{\sigma}}{T}\right)
$$

where $E_{\text {Coul }}$ is given by:

$$
E_{\text {Coul }}=\frac{\left(Z_{0}-Z\right) Z}{r_{0}\left(\left(A_{0}-A\right)^{1 / 3}+A^{1 / 3}\right)}\left(1-e^{-x \varepsilon}\right) .
$$

Here $r_{0}=1.2 \mathrm{fm}$. The term $1-e^{-x \varepsilon}$ gives an account of the Coulomb energy behavior that vanishes as $x \varepsilon$ near $T_{c}$ where no distinction exists between liquid and vapor. The fragment mass prior to decay was $A=2 Z\left(1+y\left(E^{*} / B_{f}\right)\right)$, where $B_{f}$ is the binding energy of the fragment and $y$ is a fit parameter that allows more or less decay. The temperature was determine via a degenerate Fermi gas, $T=\sqrt{E^{*} / \alpha}$, where $\alpha=8\left(1+\left(E^{*} / B_{0}\right)\right)$ [12] to accommodate the empirically observed change in $\alpha$ with $E^{*}$ [13]; $B_{0}$ is the binding energy of the fragmenting system. The total number of fragments $N_{A}$ of size $A$ was normalized to the size of the fragmenting system $A_{0}, n_{A}=N_{A} / A_{0}$.

For the ISiS data set, over 500 data points for $1.5 \leq E^{*} \leq 6.0 \mathrm{AMeV}$ and $5 \leq Z \leq 15$ were simultaneously fit to Eq. (2) with the parameters $\Delta \mu, x, \tau, \sigma, c_{0}$ and $T_{c}$ allowed to vary to minimize chi-squared. The secondary decay parameter was fixed at $y=1$. Fragments with $Z<5$ were not considered in the fit because Fisher's model expresses the 


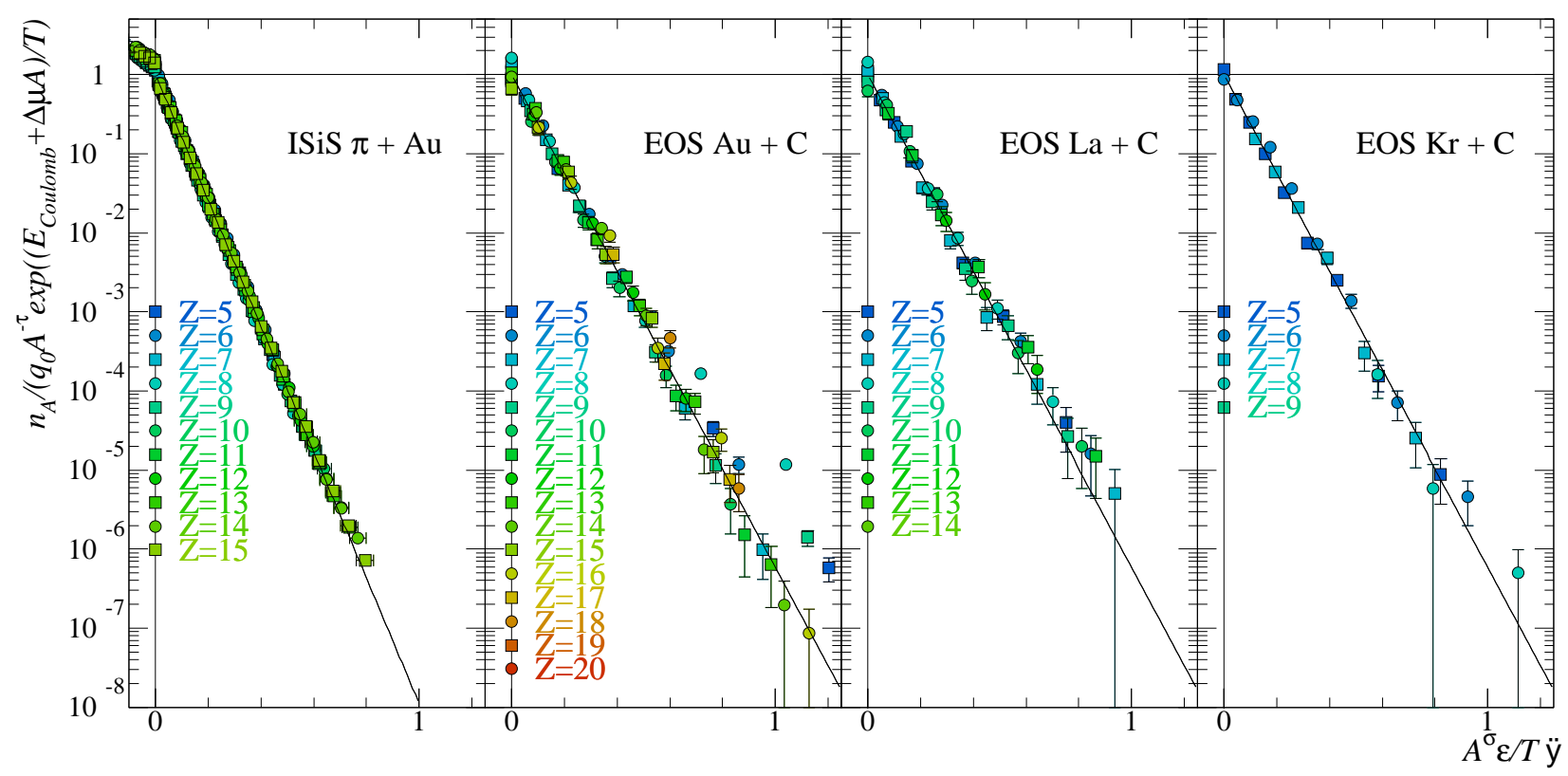

FIGURE 1. Left to right: The scaled fragment distributions of the ISiS gold data, the EOS gold, lanthanum and krypton data.

TABLE 2. Thermodynamic properties of excited nuclei

\begin{tabular}{llllllll} 
System & $E_{c}^{*}(\mathrm{AMeV})$ & $T_{c}(\mathrm{MeV})$ & $\rho_{c}\left(\rho_{0}\right)$ & $p_{c}\left(\mathrm{MeV} / \mathrm{fm}^{3}\right)$ & $\Delta H(\mathrm{MeV})$ & $\Delta E(\mathrm{AMeV})$ & $C_{c}^{F}$ \\
\hline ISiS $\pi+\mathrm{Au}$ & $3.8 \pm 0.3$ & $6.7 \pm 0.2$ & $\sim 0.3$ & $\sim 0.07$ & $26 \pm 1$ & $\sim 15$ & $0.25 \pm 0.06$ \\
EOS Au + C & $4.75 \pm 0.25$ & $7.7 \pm 0.2$ & $\sim 0.36$ & $\sim 0.11$ & $20.0 \pm 0.9$ & $\sim 11$ & $0.3 \pm 0.1$ \\
EOS La + C & $4.75 \pm 0.25$ & $7.7 \pm 0.2$ & $\sim 0.36$ & $\sim 0.11$ & $20.0 \pm 0.9$ & $\sim 11$ & $0.3 \pm 0.1$ \\
EOS Kr + C & $5.25 \pm 0.25$ & $8.2 \pm 0.2$ & $\sim 0.37$ & $\sim 0.13$ & $21.0 \pm 1.0$ & $\sim 11$ & $0.3 \pm 0.1$
\end{tabular}

mass/energy of a fragment in terms of bulk and surface energies and this approximation is known to fail for the lightest of nuclei where shell effects dominate. Aslo, for the lightest fragments equilibrium and non-equilibrium production cannot always be differentiated. Table 1 gives the resulting fit values. The value of $\tau$ and $\sigma$ are close to the values expected for some three dimensional systems: $\tau \sim 2.2$ and $\sim 2 / 3$ and are in agreement with other multifragmentation results $[14,15]$. The small positive $\Delta \mu$ value may indicate that the system is a super-saturated vapor. The value of $c_{0}$ is close to the value of the surface energy coefficient of the liquid-drop model: $16.8 \mathrm{MeV}$. The value of $T_{c}$ is close to theoretical estimates [16]. Figure 1 shows the results of this analysis: the fragment mass yields are scaled by the power law pre-factor, the bulk term and the Coulomb energy: $n_{A} / q_{0} A^{-\tau} \exp \left(\Delta \mu A+E_{C o u l} / T\right)$, and plotted against the temperature scaled by the surface energy: $A^{\sigma} \varepsilon / T$. The scaled data collapse to a single line over six orders of magnitude, precisely the behavior of a system undergoing a liquid-vapor transition. This line is the liquid-vapor coexistence line and provides direct evidence of the liquid-vapor transition in excited nuclei.

For the EOS data sets, $E_{c}^{*}$, listed in Table 2, was determined by the peak of the RMS fluctuations of the charge of the largest fragment normalized to $Z_{0}$, shown in Fig. 2. The values of $E_{c}^{*}$ are close to previous observations in the EOS data $[4,9]$ and lead to $T_{c}$ values that are comparable to theoretical estimates [16]. The topological exponent was fixed at $\tau=2.2$ in keeping with the value for a variety of three dimensional systems [17] and myriad multifragmentation studies [1,4]. There were 174 data points for $0.25 \mathrm{AMeV} \leq E^{*} \leq E_{c}^{*}$ and $5 \leq Z \leq Z_{0} / 4$ from the three data sets simultaneously fit to Eq. (2). The parameters $\sigma$ and $c_{0}$ were kept consistent between data sets while $\Delta \mu, x$ and $y$ were allowed to vary between them. The results are recorded in Table 1. The exponent values are in the range expected in Fisher's formalism for some three dimensional systems and are in agreement with those previously determined for the EOS [4] and ISiS gold multifragmentation data [15, 18], as expected for critical phenomena [19]. The surface energy coefficient $c_{0}$ is close to the value of the surface energy coefficient of the liquid-drop model. The differences in $E_{c}^{*}$ and $T_{c}$ between the ISiS and EOS data are due to the differences in differentiation between knockout particles and fragments; this difference leads to ${ }^{E O S} E^{*} \approx 1.2^{I S i S} E^{*}[8]$ which accounts for the differing results; this difference affects all energy related quantities, e.g. $c_{0}$. The larger magnitude of $\Delta \mu$ values in the EOS results compared with the 


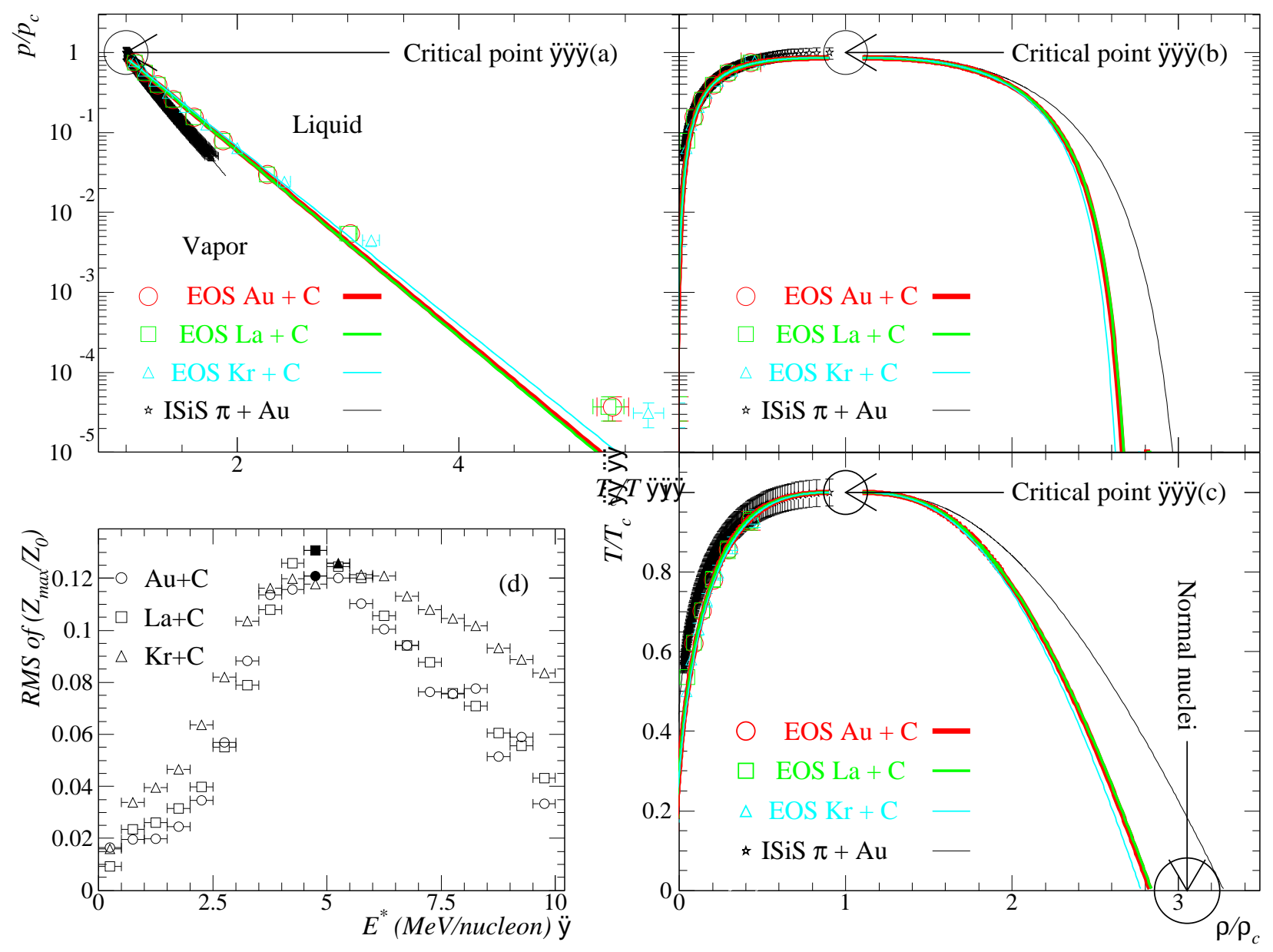

FIGURE 2. (a) The reduced pressure versus inverse reduced temperature, (b) the reduced pressure versus the reduced density and (c) the reduced temperature versus reduced density for the ISiS and EOS systems. (d) The RMS fluctuations of the charge of the largest fragment normalized to the charge of the fragmenting system versus excitation energy for the EOS systems, solid points show $E_{c}^{*}$.

ISiS results may be due to the greater degree of compression in the EOS collisions (nucleon on nucleon) compared to the ISiS collisions ( $\pi$ on nucleon). The Coulomb factor $x$ is of the same order of magnitude for both experiments. The values of $x$ may indicate more (Au and $\mathrm{La}$ ) or less $(\mathrm{Kr})$ Coulomb energy. The differences in the amount of secondary decay between the EOS and ISiS results is an open question. The EOS data scaled according to Eq. (2) shows data for all three systems collapsing onto a single line, Fig 1, illustrating the common nature of the underlying phenomenon.

Fisher assumed that a real gas of interacting particles could be treated as an ideal gas of non-interacting droplets; all of the non-ideality is accounted for in the clusterization. Thus the total pressure is found by summing the partial pressures $p / T=\sum n_{A}$ and the density is simply $\rho=\sum n_{A} A$. Accordingly, the reduced pressure is:

$$
\frac{p}{p_{c}}=\frac{T \sum n_{A}(T)}{T_{c} \sum n_{A}\left(T_{c}\right)} .
$$

The coexistence line for finite nuclear matter is obtained by using $n_{A}\left(T, \Delta \mu=0, E_{\text {Coul }}=0\right)$ from Eq. (2) in Eq. (4), transforming Fig. 1 into the familiar form shown in Fig. 2. The EOS gold and lanthanum data show nearly identical results due to their common $T_{c}$ while the krypton data differs due to it's different $T_{c}$. The different slope for the ISiS and EOS data sets is due in part to the differing energy scales. An estimate of the bulk binding energy of nuclear matter was made by recalling the Clausius-Clapeyron equation $d p / d T=\Delta H / T \Delta V$ that leads to $p / p_{c}=$ $\exp \left(\left(\Delta H / T_{c}\right)\left(1-\left(T_{c} / T\right)\right)\right)$ which describes several fluids up to $T_{c}$ [20]. The slopes of the coexistence lines and values of $T_{c}$ then give the molar enthalpy of evaporation of the liquid $\Delta H$, shown in Table 2 . The energy required to evaporate 
a fragment, the bulk binding energy, is found from $\Delta H=\Delta E+p V=\Delta E+T$, since $p V=T$ for an ideal gas. Taking into account the average fragment size along the coexistence line, $\sim 1.5$ for ISiS, $\sim 1.3$ for EOS, gives the $\Delta E /$ nucleon shown in Table 2. The value is close to the nuclear bulk energy coefficient of $15.5 \mathrm{AMeV}$. The values of $\Delta H$ and $\Delta E /$ nucleon from the ISiS data differ form those of the EOS data, due in part to the differing measures of the $E^{*}$ scale.

The reduced density of the vapor branch of the coexistence curve of finite nuclear matter is given by:

$$
\frac{\rho}{\rho_{c}}=\frac{\sum A n_{A}(T)}{\sum A n_{A}\left(T_{c}\right)} .
$$

This is shown in Fig. 2. It is possible to determine the high density branch as well: empirically, the $\rho / \rho_{c}-T / T_{c}$ coexistence curves of several fluids can be fit with: [21]

$$
\frac{\rho_{l, v}}{\rho_{c}}=1+b_{1}\left(1-\frac{T}{T_{c}}\right) \pm b_{2}\left(1-\frac{T}{T_{c}}\right)^{\beta}
$$

where the parameter $b_{2}$ is positive (negative) for the liquid $\rho_{l}$ (vapor $\rho_{v}$ ) branch. The critical exponent $\beta$ can be determined via: $\beta=(\tau-2) / \sigma$ [10]. Table 1 shows the results. Fitting the coexistence curves of the ISiS and EOS data sets with Eq. (6) gives estimates of the full $\rho_{v}$ branch of the coexistence curve. Changing the sign of $b_{2}$ gives the full $\rho_{l}$ branch of the coexistence curve of finite nuclear matter. Assuming that normal nuclei exist at the $T=0$ point of the coexistence curve in Fig. 2, then gives $\rho_{c} \sim \rho_{0} / 3$.

Dividing Eq. (4) by Eq. (5) gives the critical compressibility factor $C_{c}^{F}=p_{c} / T_{c} \rho_{c}$. Table 1 shows the results for the ISiS and EOS data which are in agreement with values of several fluids [22]. The pressure at the critical point $p_{c}$ can be found by using $T_{c}$ and $\rho_{c}$ in combination with $C_{c}^{F}$, the results are given in Table 2. This gives the a complete experimental measure of the critical point of finite nuclear matter $\left(p_{c}, T_{c}, \rho_{c}\right)$ that agrees with theoretical calculations [16]. For completeness the $p / p_{c}-\rho / \rho_{c}$ projection of the coexistence curve is determined and shown in Fig. 2.

Through a direct examination of accessible features of nuclear multifragmentation recorded by two different experiments for four different reactions a measurement of the coexistence curve of finite charged nuclear matter and estimates of the critical point have been made. The results for all systems agree, indicating that the phase diagram of nuclear matter based on experimental data has been established.

This work was supported by the US Department of Energy, the National Science Foundation, the National Science and Engineering Research Council of Canada, the Polish State Committee for Scientific Research, Indiana University Office of Research, the University Graduate School, Simon Fraser University and the Robert A. Welch Foundation.

\section{REFERENCES}

1. J. E. Finn et al., Phys. Rev. Lett. 49, 1321 (1982).

2. P. J. Siemens, Nature 305, 410 (1983).

3. L. G. Moretto et al., Phys. Rep. 287, 249 (1997).

4. J. B. Elliott et al., Phys. Rev. C 62, 064603 (2000).

5. J. Pochodzalla et al., Phys. Rev. Lett 75, 1040 (1995).

6. M. D’Agostino et al., Phys. Lett. B 473, 219 (2000).

7. K. Kwiatkowski et al., NIM A360, 571 (1995).

8. T. Lefort et al., Phys. Rev. Lett 83, 4033 (1999).

9. J. A. Hauger et al., Phys. Rev. C 62, 024626 (2000).

10. M. E. Fisher, Physics 3, 255 (1967).

11. H. Nakanishi and H. E. Stanley, Phys. Rev. B 22, 2466 (1980).

12. A.H. Raduta et al., Phys. Rev. C 55, 1344 (1997).

13. K. Hagel et al., Nucl. Phys. A486, 429 (1988).

14. M. D’Agostino et al., Nucl. Phys. A 650, 328

15. M. Kleine Berkenbusch et al., nucl-th/0109062 (2001).

16. J. N. De et al., Phys. Rev. C 59, R1 (1999).

17. D. Stauffer and A. Aharony, "Introduction to Percolation Theory", 2nd ed. (Taylor and Francis, London, 2001).

18. J. B. Elliott et al., to be published in Phys. Rev. Lett. (2001).

19. J. M. Yeomans, "Statistical Mechanics of Phase Transitions", 1rst ed. (Clarendon Press, Oxford 1992).

20. E.A. Guggenheim, "Thermodynamics”, 4th ed. (North-Holland, 1993).

21. E. A. Guggenheim, J. Chem. Phys., 13, 253 (1945).

22. C. S. Kiang, Phys. Rev. Lett. 24, 47 (1970). 